ISSN 2075-1680

www.mdpi.com/journal/axioms

Article

\title{
Second-Order Risk Constraints in Decision Analysis
}

\section{Love Ekenberg $^{1, *}$, Mats Danielson ${ }^{1}$, Aron Larsson ${ }^{1,2}$ and David Sundgren ${ }^{1}$}

${ }^{1}$ Department of Computer and Systems Sciences, Stockholm University, Kista 164 40, Sweden; E-Mails: mad@dsv.su.se (M.D.); aron@dsv.su.se (A.L.); dsn@dsv.su.se (D.S.)

${ }^{2}$ Risk and Crisis Research Center, Mid Sweden University, Sundsvall 851 70, Sweden

* Author to whom correspondence should be addressed; E-Mail: lovek@dsv.su.se;

Tel.: +46-8-703-90-25.

Received: 8 November 2013; in revised form: 24 December 2013 / Accepted: 27 December 2013 / Published: 17 January 2014

\begin{abstract}
Recently, representations and methods aimed at analysing decision problems where probabilities and values (utilities) are associated with distributions over them (second-order representations) have been suggested. In this paper we present an approach to how imprecise information can be modelled by means of second-order distributions and how a risk evaluation process can be elaborated by integrating procedures for numerically imprecise probabilities and utilities. We discuss some shortcomings of the use of the principle of maximising the expected utility and of utility theory in general, and offer remedies by the introduction of supplementary decision rules based on a concept of risk constraints taking advantage of second-order distributions.
\end{abstract}

Keywords: decision analysis; second-order information; risk analysis; risk constraints

\section{Introduction}

Methods and tools for analyzing and evaluating decision problems under risk have been of great interest for a long time. During recent decades, such methods have been more or less systematically integrated with risk management processes in general [1-3] and, in more concrete settings such as project management frameworks, with risk identification, monitoring, and evaluation needs [4]. The prevailing decision rule serving as an instrument for ensuring substantial rationality in decision making under risk is commonly referred to as the principle of maximizing the expected utility (PMEU). The principle is inspired by early efforts in normative decision theory, e.g., [5-7]. It is derived from a number of different, 
although similar, axiom systems aiming to reflect the properties of a rational entity's behavior when discriminating between decision alternatives given that the consequences of an alternative are uncertain but the set of possible consequences for each alternative can be assigned performance numbers (utilities) and probabilities of their occurrence. The initial formal verification of this rule is commonly credited to [5] and was initially intended as a model of the supposedly rational market actor's behavior in the game theory school of economic thought. The axioms (ordering axioms, independence axioms, continuity axioms, etc.) thus imply numerical representations of preferences and probabilities. Further, implied by the axioms are existence theorems stating that a utility function exists and a uniqueness theorem stating that two utility functions, relative to a given preference ranking, are always affine transformations of each other. It is often argued that these results provide justification of the PMEU.

However, the conclusion that the PMEU is logically sufficient given the axioms, and whether or not the axioms themselves reflect the properties of a rational decision-maker, has not passed without criticism and debate. For instance, in [8] it is shown in a formal investigation that the relation between the utility principle and the axioms is not as strong as claimed, but rather that "an agent who endorses these axioms is not contradicting himself if he also accepts the utility principle" and that the PMEU is the simplest principle that is consistent with the axioms. Further, the use of a utility function for capturing all possible risk attitudes is not considered possible [9].

As a result, some researchers have tried to modify the application of PMEU by bringing regret or disappointment into the evaluation to cover cases where numerically equivalent results are appreciated differently depending on what was once in someone's possession, e.g., [10]. Others have tried to resolve the problems mentioned above by having functions modifying both the probabilities and the utilities, but their performances are at best equal to that of the expected value, and at worst inferior, e.g., inconsistent with first-order stochastic dominance [11].

An important issue in PMEU-based decision analysis is the elicitation of a decision-maker's attitude towards risk. However, the elicitation of risk attitudes from human decision-makers is error prone and the result is highly dependent on the formats and methods used, see, e.g., [12,13] for overviews of the state-of-the-art in elicitation. This problem is even more evident when the decision situation involves catastrophic outcomes [14]. If we are not able to elicit a properly reflecting risk attitude, we may have the situation that even if the evaluation of an alternative results in an acceptable expected utility, some consequences might be of a catastrophic kind so that the alternative should be avoided in any case. Due to catastrophe aversion, this may be the case even if the probabilities of these consequences are very low. In such cases, the PMEU needs to be extended with other rules, since not all risk behaviors can naturally be modeled endogenously by a utility function in the traditional way using the classical notion of risk averseness and proneness.

Within the decision analysis field, having a more pragmatic approach than a purely normative theory of rational choice, the PMEU is most often deemed as sufficient in order to serve as a valuable tool for comparing decision alternatives, see, e.g., [15]. Also, in [11] the performance of a number of various decision rules is investigated, including that of [10], and it is concluded that from a decision analysis perspective there is really no other rule better suited to serve as the underlying basic decision rule.

In the context of catastrophic events, the partitioned multi-objective risk method (PMRM) and the use of conditional expected utilities for the modeling of decision problems in low-likelihood 
severe-consequence domains have been suggested [16,17]. This approach puts emphasis on the tails of probability distributions over different kinds of values at risk in the case of a catastrophic scenario; still not discarding the unconditional probability of an extreme event actually occurring, but treating both the conditional and unconditional expected values as decision objectives. The approach outlined in this paper is nevertheless related to conditional expected utility approaches toward decision analysis in the face of extreme events, since we are concerned with consequences that have a very low probability of actually occurring. However, although there is no rule deemed to be better than the PMEU, there is a need to allow the use of complementary rules in applications of decision analysis; rules also acknowledging that decision data often is subject to imprecision. It is argued that a useful decision theory should permit a wider spectrum of the modeling of risk attitudes than merely by means of a single utility function. A more pragmatic approach should give the decision-maker the means to express risk attitudes in a variety of ways, as well as provide procedures for handling both qualitative (e.g., comparisons) and quantitative (e.g., intervals) aspects.

We will now take a closer look at such an approach, where some of the inherent deficiencies are remedied in a decision analytical context. The approach relies on a traditional decision tree model, however allowing for interval statements of probabilities and utilities together with associated second-order distributions. The next section introduces a decision tree formalism and corresponding risk constraints, followed by a brief description of a theory for representing imprecision using second-order distributions. The last section before the conclusion presents how risk constraints can be realized in a second-order framework for evaluating decisions under risk together with a small example using two approaches to cope with imprecise information when evaluating decision alternatives with risk constraints.

\section{Modeling the Decision Problem}

We will let an information frame represent a decision problem. The purpose of such a frame is to collect all information necessary for the model into one structure. The representations in the information frame are of two kinds; a decision structure, modeled by means of a conventional decision analysis decision tree, i.e., a graph structure $\langle V, E\rangle$ where $V$ is a set of nodes and $E$ is a set of node pairs (edges). Each node is at a specific level, numbered from the top node which is at level 0 . We also have input statements, modeled by linear constraints.

Definition 1. A tree is a connected graph without cycles. A decision tree is a tree containing a finite set of nodes which has a dedicated node at level 0 . A node at level $i+1$ that is adjacent to a node at level $i$ is a child of the latter. A path leading to a node at level 1 is an alternative. A node at level $i$ is a leaf or consequence if it has no adjacent nodes at level $i+1$. A node that is at level 2 or more and has children is an event (an intermediary node). The depth of a rooted tree is $\max (n \mid$ there exists a node at level $\mathrm{n}$ ). See Figure 1 for an example of a decision tree.

Thus, a decision tree is a way of modeling a decision situation. In risk analysis practice, such trees with sequences of events are often referred to as event trees used to describe accident sequences [18]. For convenience we use the notation that the $n$ children of a node $x_{i}$ are denoted $x_{i 1}, x_{i 2}, \ldots, x_{i n}$ and the 
$m$ children of the node $x_{i j}$ are denoted $x_{i j 1}, x_{i j 2}, \ldots, x_{i j m}$ and so forth. For presentational purposes, we will denote a consequence node of an alternative $A_{i}$ simply with $C_{i j}$.

Figure 1. Small decision tree.

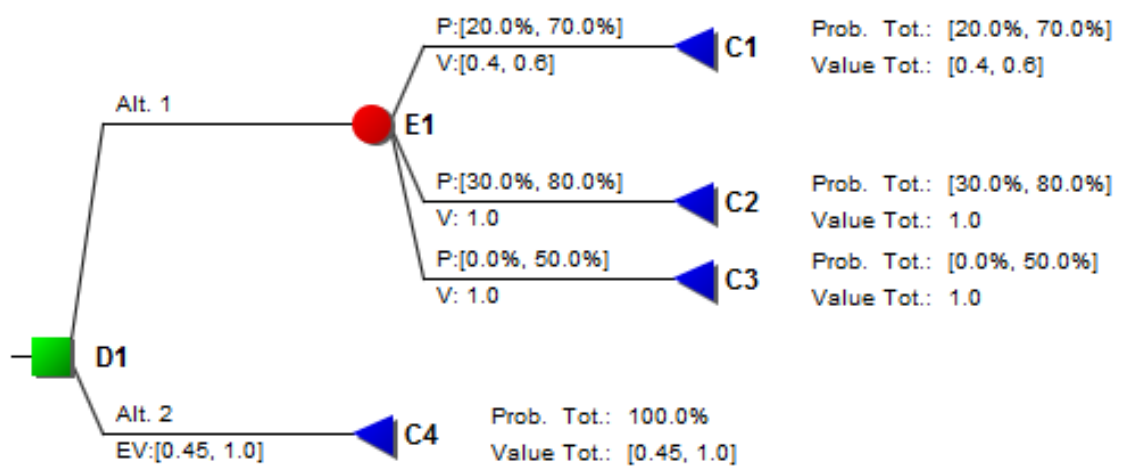

In numerically imprecise decision situations, one widespread modeling approach is to define sets of candidates of possible probability distributions over the event nodes and utility functions over the consequence nodes and then express them as points in polytopes that are solution sets to probability and utility bases [19]. For instance, the probability (or utility) of $C_{i j}$ being between the numbers $a_{k}$ and $b_{k}$ is expressed as $p_{i j} \in\left[a_{k}, b_{k}\right]$ (or $u_{i j} \in\left[a_{k}, b_{k}\right]$ ). Such an approach also includes relations: a measure (or function) of $C_{i j}$ is greater than a measure (or function) of $C_{k l}$ is expressed as $p_{i j} \geq p_{k l}$ and analogously $u_{i j} \geq u_{k l}$. Each statement can thus be represented by one or more constraints.

Definition 2. Given a decision tree $T$, a utility base is a set of linear constraints of the types $u_{i j} \in\left[a_{k}, b_{k}\right], u_{i j} \geq u_{k l}$ and, for all consequences $\left\{C_{i j}\right\}$ in $T, u_{i j} \in[0,1]$. A probability base has the same structure, but for an intermediate node $N$ (except the root node) in $T$, it also includes $\sum_{j=1}^{m_{N}} p_{i j}=1$ for the children $\left\{x_{i j}\right\}_{j=1, \ldots, m_{N}}$ of $N$.

The solution sets to probability and utility bases are polytopes in hypercubes. Since a point in the polytope can be considered to represent a distribution, a probability base $\mathcal{P}_{i}$ can be interpreted as constraints defining the set of all possible probability measures over the consequences. Similarly, a utility base $\mathcal{U}$ consists of constraints defining the set of all possible utility functions over the consequences. The union of the bases $\mathcal{P}_{i}$ and $\mathcal{U}$ together with the decision tree constitute the information frame $\mathcal{I}=\langle T, \mathcal{P}, \mathcal{U}\rangle$, where $\mathcal{P}=\bigcup \mathcal{P}_{i}$. As discussed above, the most common evaluation rules of a decision tree model are based on the PMEU [20].

Definition 3. Given an information frame $\mathcal{I}=\langle T, \mathcal{P}, \mathcal{U}\rangle$ and an alternative $A_{i} \in A$ the expression

$$
E\left(A_{i}\right)=\sum_{i_{1}=1}^{n_{i_{0}}} p_{i i_{1}} \sum_{i_{2}=1}^{n_{i_{1}}} p_{i i_{1} i_{2}} \ldots \sum_{i_{m-1}=1}^{n_{i_{m-2}}} p_{i i_{1} i_{2} \ldots i_{m-2} i_{m-1}} \sum_{i_{m}=1}^{n_{i_{m-1}}} p_{i i_{1} i_{2} \ldots i_{m-2} i_{m-1} i_{m}} u_{i i_{1} i_{2} \ldots i_{m-2} i_{m-1} i_{m}}
$$

is the expected utility of alternative $A_{i}$ in $\mathcal{I}$, where $m$ is the depth of the tree corresponding to $A_{i}$, $n_{i_{k}}$ is the number of possible outcomes following the event with probability $P\left(x_{i_{k}}\right)=p_{i_{k}}, p_{\ldots i_{j} \ldots}, j \in$ $[1, \ldots, m]$, denoting probability variables and $u_{\ldots i_{j} \ldots}$ denoting utility variables as above. 
The alternatives in the tree are evaluated according to PMEU, and the resulting expected utilities yield a preference ordering of the alternatives such that $A_{i}$ is not preferred to $A_{j}$ if and only if $E\left(A_{i}\right) \leq E\left(A_{j}\right)$. Note that interval statements can yield a partial order due to overlapping expected utility intervals. However, as discussed in the introduction, the use of utility functions to formalize the decision process seems to be an oversimplified idea, disregarding factors typically present in real-life applications of decision analysis. Therefore, there is a need to permit the use of additional ways to discriminate between alternatives. The next section discusses risk constraints as such an additional decision rule.

\section{Risk Constraints}

The intuition behind risk constraints is that they state when an alternative is undesirable due to too risky consequences. It is intended as a pragmatic approach to model aversion to catastrophes in decision analysis applications, and builds upon the idea of providing thresholds beyond which an alternative is deemed undesirable by a decision-maker. Such assessments are common in risk management processes, where risk is typically linked to the probability of some event or consequence which is found to be undesirable [21]. Thus, expressing risk constraints is analogous to expressing minimum requirements that should be fulfilled. A risk constraint can be viewed as a function yielding a set of thresholds that may not be violated in order for an alternative to be acceptable with respect to risk [22].

Thus, a decision-maker might regard an alternative as undesirable if it has consequences with too low a utility and with some probability of occurring, even though those particular consequences' contributions to the expected utility are low. This mechanism is fairly straightforward. Assuming a 1-level tree, an alternative $A_{i}$ in an information frame $\mathcal{I}$, and given a utility threshold $r$ and a probability threshold $s$, the inequality

$$
\sum_{u_{i j} \leq r} p_{i j} \leq s
$$

must be satisfied in order for $A_{i}$ to be deemed an acceptable alternative. In this sense, a risk constraint can be considered a utility-probability pair $(r, s)$. A consequence $C_{i j}$ is said to be violating $r$ if $u_{i j}>r$ does not always hold. Principles of this kind seem to be good prima facie candidates for evaluative principles in the literature, i.e., they conform well to established practices and enable a decision-maker to use qualitative assessments in a reasonable way. For a comprehensive treatment and discussion, see $[23,24]$.

Note that henceforth we will, without loss of generality, consider 1-level trees. An $n$-level tree can always be collapsed into a 1-level counterpart. In the case when there are second-order distributions over the intervals, the process of collapsing trees become more complicated and is treated in Section 4.2 below.

When the information is numerically imprecise (i.e., probabilities and utilities are expressed as bounds or intervals), it is not obvious how to interpret thresholds since a risk constraint may cease to be violated in sub-sets of the solution set. We have earlier suggested that the interval boundaries together with stability analyses could be considered in these cases [25].

Example 1. Assume that a decision-maker has asserted that an alternative $A_{i}$ is considered undesirable if the consequence $C_{i j}$ belonging to $A_{i}$ has a possibility that the utility of $C_{i j}$ is less than 0.45 , and if the 
probability of $C_{i j}$ is greater than 0.65 . Furthermore, assume that alternative $A_{1}$ has a consequence with a utility in the interval $[0.40,0.60]$. Further assume that the probability of this consequence lies in the interval $[0.20,0.70]$ and that the minimum of all utilities of consequences of $A_{2}$ are above 0.45 . Since 0.45 is greater than the least possible utility of the consequence and 0.65 is less than the greatest possible probability, then $A_{1}$ violates the thresholds and is undesirable, while $A_{2}$ is not, see Figure 2.

Figure 2. Contraction analysis of risk constraints given in Example 1. Beyond a contraction level of $14 \%$, the constraints are no longer violated for alternative $A_{1}$. The constraints for alternative $A_{2}$ are never violated. A decision making agent might nevertheless be inclined to accept the alternative since the constraints are violated in a small enough proportion of the possible values.

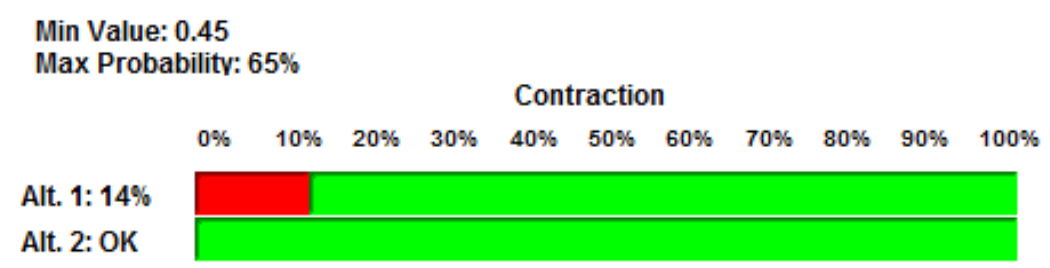

For a stability analysis, it can be seen that the alternative in Example 1 ceases to be undesirable when the left end-point of the utility interval is increased by 0.05 . A concept in line with such stability analyses is the concept of interval contraction, investigating to what extent the widths of the input intervals need be reduced in order for an alternative not to violate the risk constraints. The contractions of intervals are made toward a contraction point for each interval. Contraction points can either be given explicitly by the decision-maker or be suggested from, e.g., centre of mass calculations. The level of contraction is indicated as a percentage, where at $100 \%$ contraction all intervals have been replaced with their contraction points. See Figure 2 for a contraction analysis of the rudimentary problem in Example 1.

Thus, one refinement is to provide a possibility for a decision-maker to stipulate thresholds for proportions or second-order probabilities of the probability and utility bases, i.e., an alternative is considered unacceptable if it violates the risk constraints at a given contraction level [22,26]. Optionally, an alternative is unacceptable if the probability of violating a risk constraint is above a certain threshold. That is, alternatives are judged by the risk of violating risk constraints where the decision rule is risk of the second order. Since some contractions (lowering of some upper probability bounds and increasing lower utility bounds) decrease the probability of risk constraint violation (see Section 5), the two views of a second-order risk based decision rule are closely related.

\section{Second-Order Information}

In interval-valued decision trees, the expected utility of an alternative will also become interval-valued. In real-life cases it is then often hard to discriminate between the alternatives since the intervals overlap. For instance, an interval-based decision procedure keeps all alternatives with overlapping expected utility intervals, even if the overlap is small. Therefore, it is worthwhile to extend 
the representation of the decision situation using more information, such as second-order distributions over classes of probability and utility measures.

Distributions can be used for expressing various beliefs over multi-dimensional spaces where each dimension corresponds to possible probabilities or utilities of consequences. The distributions can be used to express strengths of belief in different points in the polytopes. Approaches for extending the interval representation using distributions over classes of probability and value measures have been developed into various models, for instance second-order probability theory. In the following, we will pursue the idea of adding more information and discuss its implications on risk constraints.

\subsection{Distributions over Information Frames}

Interval estimates and relations can be considered as special cases of representations based on distributions over polytopes. For instance, a distribution can be defined to have a positive support only for $x_{i} \leq x_{j}$. More formally, the solution set to a probability or utility base is a subset of a unit cube since both variable sets have $[0,1]$ as their ranges. This subset can be represented by the support of a distribution over the cube.

Definition 4. Let a unit cube $[0,1]^{n}$ be represented by $B=\left(b_{1}, \ldots, b_{n}\right)$. The $b_{i}$ can be explicitly written out to make the labeling of the dimensions clearer.

More rigorously, the unit cube is represented by all the tuples $\left(x_{1}, \ldots, x_{n}\right)$ in $[0,1]^{n}$.

Definition 5. A second-order distribution over a unit cube $B$ is a positive distribution $F$ defined on $B$ such that

$$
\int_{B} F(x) d V_{B}(x)=1
$$

where $V_{B}$ is the $n$-dimensional Lebesgue measure on $B$.

We consider second-order probabilities to be an important sub-class of these distributions. Second-order probabilities will be used below as a measure of belief, i.e., a second-order joint probability distribution. Such distributions can then be defined over the information frame polytopes. However, regardless of the actual shapes of the distributions involved, constraints such as $\sum_{i=1}^{n} x_{i}=1$ must be satisfied since it is not reasonable to believe in an incoherent probability distribution over three mutually exclusive outcomes such as $(0.45,0.25,0.4)$. For this purpose, any multivariate probability distribution with support on vectors of non-negative variables that sum to one can serve as second-order distribution. However, a particularly suitable way of modeling random probabilities is the Dirichlet distribution.

Definition 6. Let the notation be as above. Then the probability density function of the Dirichlet distribution is defined as

$$
f_{\text {Dir }}(p, \alpha)=\frac{\Gamma\left(\sum_{i=1}^{n} \alpha_{i}\right)}{\prod_{i=1}^{n} \Gamma\left(\alpha_{i}\right)} p_{1}^{\alpha_{1}-1} p_{2}^{\alpha_{2}-1} \cdots p_{n}^{\alpha_{n}-1}
$$

on a set $\left\{p=\left(p_{1}, \ldots, p_{n}\right) \mid p_{1}, p_{2}, \ldots, p_{n} \geq 0, \sum p_{i}=1\right\}$, where $\left(\alpha_{1}, \alpha_{2}, \ldots, \alpha_{n}\right)$ is a parameter vector in which each $\alpha_{i}$ is a positive parameter and $\Gamma\left(\alpha_{i}\right)$ is the Gamma function. 
This distribution is particularly popular among Bayesian statisticians because it is conjugate with respect to the multinomial distribution, i.e., if we choose the prior to be the Dirichlet distribution then the posterior will also become Dirichlet distributed. It is also convenient in the sense that it is not hard to choose parameters to reflect our prior knowledge about the weights $p_{1}, p_{2}, \ldots, p_{n}$. If we choose large values for $\alpha_{1}, \alpha_{2}, \ldots, \alpha_{n}$ we obtain small variances, which reflect a large measure of certainty about the probabilities involved.

If the support of a probability density function is restricted to a subset $A$ of a unit cube $B$, for instance when $A$ is a polytope within $B$ having the same number of dimensions as $B$, beliefs in different points or subsets can be represented by a function defined on the specific subset. However, if we want to represent the belief on a subset which is of lower dimension than the unit cube itself we cannot use distributions that are upper bounded since a mass under such a distribution will be 0 while integrating with respect to the Lebesgue measure defined on the unit cube. This issue can be solved by the characteristic distribution for $A$.

Definition 7. Let $A$ be a subset of a unit cube $B$, and let $f$ be a belief distribution over $A$. The natural extension $\tilde{f}_{A}(x)$ of $f$ with respect to $A$ is defined by

$$
\tilde{f}_{A}(x)= \begin{cases}f(x) & \text { if } x \in A \\ 0 & \text { otherwise }\end{cases}
$$

Definition 8. Let $A$ be a subset of $B$. A distribution $g_{A}$ over $B$ is called a characteristic distribution for $A$ in $B$ if

$$
f(p)=\int_{B} \delta_{p}(x) \tilde{f}_{A}(x) g_{A}(x) d V_{B}(x)
$$

for every probability density function $f$ over $A$, and for every point $p$ in $A$, where $\delta_{p}(x)$ is the Dirac delta distribution with pole at $p$.

For a more comprehensive treatment of these properties, see [27]. With respect to second-order probabilities, let $A=\left\{\left(p_{1}, \ldots, p_{n}\right) \mid \sum_{i=1}^{n} p_{i}=1\right\}$ and let $g_{A}$ be a Dirichlet distribution. From distribution theory it follows that for every measurable subset $A$ in a unit cube $B$, there exists a characteristic distribution for $A$ in $B$. It also follows that $\tilde{f}_{A}(x) \cdot g_{A}(x)$ is a probability distribution over $B$ and equals 0 outside $A$.

\subsection{Aggregations and Expected Utility}

The characteristic of a decision tree is that the marginal (or conditional) probabilities of the event nodes are multiplied in order to obtain the joint probability of a combined event, i.e., of a path from the root to a leaf. In the evaluation of a decision tree by means of PMEU the operations involved are multiplications and additions. There are therefore two effects present at the same time when calculating expected utilities in decision trees. Those are additive effects (for joint probabilities aggregated together with the utilities at the leaf nodes) and multiplicative effects (for intermediate probabilities). The next section discusses how second-order information may be exploited for risk constraints. 


\section{Second-Order Risk Constraints}

Given first-order risk constraints we can consider the probability that input statements support a violation of a risk constraint $(r, s)$ for a given alternative $A_{i}$. The value of this probability delivers further information to a decision-maker when more than one alternative violates stipulated risk constraints. This is especially important for cases when only some consistent probability-utility assignments (i.e., subsets of the polytopes) violate the risk constraints.

If an alternative does not violate the first-order risk constraint for any consistent (first-order) probabilities or utilities in the information frame, then the probability of a violation is zero. On the other hand, if all consistent probabilities and utilities violate the risk constraint, the probability of violation equals one. We will now extend the discussion to second-order risk constraints (SORC).

Begin by defining $B_{R}=B_{P_{i}} \times B_{U_{i}}$, consisting of all tuples $(p, u)$, i.e., $\left(p_{i 1}, u_{i 1}, \ldots, p_{i n}, u_{i n}\right)$. Let $F_{i}$ be a second-order probability distribution on $B_{P_{i}}$ and let $G_{i}$ be a probability distribution on $B_{U_{i}}$.

Given an information frame $\mathcal{I}$, if probabilities and utilities are independent, the joint event that probabilities have values $p$ and utilities have values $u$ has the second-order probability $F_{i}(p) \cdot G_{i}(u)$.

In Section 3 a risk constraint $(r, s)$ is said to be violated if $\sum_{j \mid u_{i j}<r} p_{i j}>s$. In a second-order setting the precise values of probabilities $p$ and utilities $u$ are not known but random variables. Thus, to extend the notion of risk constraint violation to account for second-order beliefs we should weigh the probabilities and utilities by their respective probability distributions in the manner of a cumulative probability distribution. Let

$$
\tau_{(\mathcal{I}, i, r, s)}=\int_{B_{R}} H_{i}(p, u) d V_{B_{R}}(p, u)
$$

where

$$
H_{i}(p, u)= \begin{cases}F_{i}(p) \cdot G_{i}(u) & \text { if } \sum_{j \mid u_{i j}<r} p_{i j}>s \\ 0 & \text { otherwise }\end{cases}
$$

is the second-order probability that first-order probabilities and utilities are such that risk constraint $(r, s)$ is violated. This is called a constraint violation.

We will now show that the concept of SORC fulfils some fundamental requirements.

Theorem 1. Given an information frame $\mathcal{I}=\langle T, \mathcal{P}, \mathcal{U}\rangle$, alternative $A_{i}$, utility threshold $r$ and probability threshold $s, \tau_{(\mathcal{I}, i, r, s)}$ fulfills requirements $1-6$.

1. Complementary cumulative distribution function. $\tau_{(\mathcal{I}, i, r, s)}$ is the probability that $\sum_{j \mid u_{i j}<r} p_{i j}$ exceeds $s$. In other words, $\tau_{(\mathcal{I}, i, r, s)}$ is the complementary cumulative distribution function of the random variable $\sum_{j \mid u_{i j}<r} p_{i j}$.

2. Utility Sharpening. Given risk constraints $\left(r_{1}^{\prime}, s\right),\left(r_{2}^{\prime}, s\right)$, and an information frame $\mathcal{I}$ with an alternative $A_{i}$. Then $r_{1}^{\prime}>r_{2}^{\prime} \Rightarrow \tau_{\left(\mathcal{I}, i, r_{1}^{\prime}, s\right)} \geq \tau_{\left(\mathcal{I}, i, r_{2}^{\prime}, s\right)}$.

3. Probability Sharpening. Given risk constraints $\left(r, s_{1}^{\prime}\right),\left(r, s_{2}^{\prime}\right)$, and an information frame $\mathcal{I}$ with an alternative $A_{i}$. Then $s_{1}^{\prime}<s_{2}^{\prime} \Rightarrow \tau_{\left(\mathcal{I}, i, r, s_{1}^{\prime}\right)} \geq \tau_{\left(\mathcal{I}, i, r, s_{2}^{\prime}\right)}$. 
4. Utility Contraction. If utility $u_{i j}$ is contracted so that $u_{i j} \geq l_{i j}^{\prime}>l_{i j}$, i.e., $u_{i j}$ had lower bound $l_{i j}$ originally but $l_{i j}^{\prime}$ after contraction, and the corresponding information frames are the original $\mathcal{I}$ and the contracted $\mathcal{I}^{\prime}$ then $\tau_{(\mathcal{I}, i, r, s)} \geq \tau_{\left(\mathcal{I}^{\prime}, i, r, s\right)}$.

5. Probability Contraction. If probability $p_{i j}$ is contracted so that $p_{i j} \leq m_{i j}^{\prime}<m_{i j}$, i.e., $p_{i j}$ had upper bound $m_{i j}$ originally but $m_{i j}^{\prime}$ after contraction, and the corresponding information frames are the original $\mathcal{I}$ and the contracted $\mathcal{I}^{\prime}$ then $\tau_{(\mathcal{I}, i, r, s)} \geq \tau_{\left(\mathcal{I}^{\prime}, i, r, s\right)}$.

6. Addition of Risky Consequence. Given risk constraint $(r, s)$, an information frame $\mathcal{I}_{1}$ with an alternative $A_{i}$ and a consequence $C_{i k}=\left(p_{i k}, u_{i k}\right)$ such that $\left(p_{i 1}, u_{i 1}, \ldots, p_{i n}, u_{i n}\right)$ does not violate $r$. Let $\mathcal{I}_{2}$ be another information frame identical to $\mathcal{I}_{1}$ except for that $C_{i k}$ is such that $\left(p_{i 1}, u_{i 1}, \ldots, p_{i n}, u_{i n}\right)$ does violate $r$. Then $\tau_{\left(\mathcal{I}_{2}, i, r, s\right)} \geq \tau_{\left(\mathcal{I}_{1}, i, r, s\right)}$.

Proof. 1. As before, let $p$ and $u$ be independent random variables with probability densities $F_{i}(p)$ and $G_{i}(u)$ respectively. Let $f_{\Sigma}$ be an integral such that

$$
f_{\Sigma}(t)=\int_{C(t)} F_{i}(p) \cdot G_{i}(u) d V_{B_{R}}(p, u)
$$

where

$$
C(t)=\left\{(p, u) \in B_{R} \mid \sum_{j \mid u_{i j}<r} p_{i j}=t\right\}
$$

Then $f_{\Sigma}$ is a probability density function over the random variable $\sum_{j \mid u_{i j}<r} p_{i j}$. First, since $F_{i}(p)$ and $G_{i}(u)$ are probability density functions, the integrand $F_{i}(p) \cdot G_{i}(u) \geq 0$, hence

$$
\int_{C(t)} F_{i}(p) \cdot G_{i}(u) d V_{B_{R}}(p, u) \geq 0
$$

Secondly, since $p$ and $u$ are independent, the joint density of $(p, u)$ is $F_{i}(p) \cdot G_{i}(u)$, hence

$$
\int_{B_{R}} F_{i}(p) \cdot G_{i}(u) d V_{B_{R}}(p, u)=1
$$

However, $\int_{-\infty}^{\infty} f_{\Sigma}(t) d t=\int_{0}^{1} f_{\Sigma}(t) d t$ since $0 \leq \sum_{j \mid u_{i j}<r} p_{i j} \leq 1$, and the set $B_{R}$ of $(p, u)$-pairs is partitioned by the relation that $\sum_{j \mid u_{i j}<r} p_{i j}$ is equal, hence

$$
\int_{0}^{1} f_{\Sigma}(t) d t=\int_{0}^{1} \int_{C(t)} F_{i}(p) \cdot G_{i}(u) d V_{B_{R}}(p, u) d t=\int_{B_{R}} F_{i}(p) \cdot G_{i}(u) d V_{B_{R}}(p, u)=1
$$

Let $A=\left\{(p, u) \in B_{R} \mid \sum_{j \mid u_{i j}<r} p_{i j}>s\right\}$. Now we can see that $\tau_{(\mathcal{I}, i, r, s)}$ is the complementary cumulative distribution function of $\sum_{j \mid u_{i j}<r} p_{i j}$, i.e., $\tau_{(\mathcal{I}, i, r, s)}=\int_{s}^{1} f_{\Sigma}(t) d t$ since

$$
\begin{array}{r}
\tau_{(\mathcal{I}, i, r, s)}=\int_{B_{R}} H_{i}(p, u) d V_{B_{R}}(p, u)=\int_{A} F_{i}(p) \cdot G_{i}(u) d V_{B_{R}}(p, u)= \\
\int_{s}^{\infty} \int_{C(t)} F_{i}(p) \cdot G_{i}(u) d V_{B_{R}}(p, u) d t=\int_{s}^{1} f_{\Sigma}(t) d t
\end{array}
$$


2. We have risk constraints $\left(r_{1}^{\prime}, s\right)$ and $\left(r_{2}^{\prime}, s\right)$. As the proportion of points $(p, u) \in B_{R}$ violating $(r, s)$ must be bounded above by $u_{i j} \leq r, r_{1}^{\prime}>r_{2}^{\prime}$ cannot result in a lower proportion for $r_{1}^{\prime}$ than for $r_{2}^{\prime}$.

3. Is shown in the same way.

4. The same proof idea as for 2 applies also here; with a new lower bound $l_{i j}^{\prime}$ the proportion of points $(p, u) \in B_{R}$ violating $(r, s)$ can be no higher than it is with the lower bound $l_{i j}<l_{i j}^{\prime}$.

5. The proportion of points $(p, u) \in B_{R}$ violating $(r, s)$ when $p_{i j} \leq m_{i j}^{\prime}$ can be no higher than with the upper bound $m_{i j}>m_{i j}^{\prime}$.

6. We have two initial cases. For the first case when $\sum_{j \mid u_{i j}<r} p_{i j} \leq s$ always hold given $\mathcal{I}_{2}$, then it must always hold given $\mathcal{I}_{1}$ and $H_{i}(p, u)$ is zero everywhere for both information frames and in the first case $\tau_{\left(\mathcal{I}_{1}, i, r, s\right)}=\tau_{\left(\mathcal{I}_{2}, i, r, s\right)}=0$. For the second case, when $\sum_{j \mid u_{i j}<r} p_{i j} \leq s$ does not always hold given $\mathcal{I}_{2}$, then $H_{i}(p, u)$ has positive support and $\tau_{\left(\mathcal{I}_{2}, i, r, s\right)}>0$. Since $C_{i k}$ did not violate $r$ given $\mathcal{I}_{1}$, the proportion of points $(p, u) \in B_{R}$ where $H_{i}(p, u)>0$ is smaller for $\mathcal{I}_{1}$ compared to for $\mathcal{I}_{2}$. Thus in the second case $\tau_{\left(\mathcal{I}_{2}, i, r, s\right)}>\tau_{\left(\mathcal{I}_{1}, i, r, s\right)} \geq 0$.

These properties make SORC a useful tool for handling risks in (second-order) decision analysis.

\section{Examples}

To illustrate how second-order risk constraints contrast with first-order models and how SORC can show distinctions that are not possible to reveal with first-order models such as contraction we show two examples.

Example 2. Consider the decision tree in Figure 1 with risk constraints $(r, s)=(0.45,0.65)$, i.e., equal to Example 1. Then $A_{1}$ violates these risk constraints. From the contraction analysis in Example 1 we know that the risk constraints are not violated for every point in the solution set. In fact, from simulation it can be seen that the violation belief is approximately $0.25 \%$. A decision-maker could therefore more confidently accept the alternative since the constraints are violated in small proportion, see Figure 3.

Figure 3. Second-order analysis of risk constraints given in Example 2. Although we needed a contraction level of $14 \%$ in order to not violate the risk constraints, the violation belief is merely about $0.25 \%$.

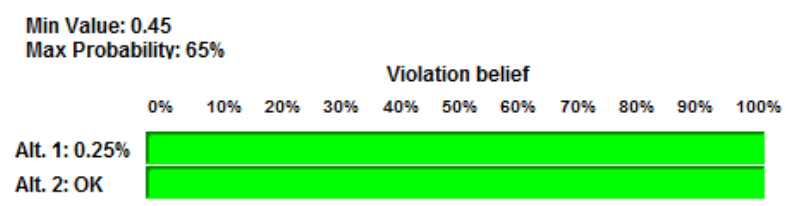

Example 3. Consider the decision tree in Figure 4 with two alternatives each having eight uncertain consequences. Both $A_{1}$ (upper branch) and $A_{2}$ (lower branch) clearly violate these risk constraints $(0.1,0.1)$. From a contraction analysis, it can be seen that the risk constraints cease to be violated at a 
contraction level of $19 \%$ for both alternatives. However, the violation belief is larger for $A_{2}$ compared to $A_{1}$. Thus, contraction analysis and similar means for sensitivity analysis in interval decision analysis is less responsive to the fundamental requirement of 3 , the probability sharpening condition.

Figure 4. Decision tree in Example 3.

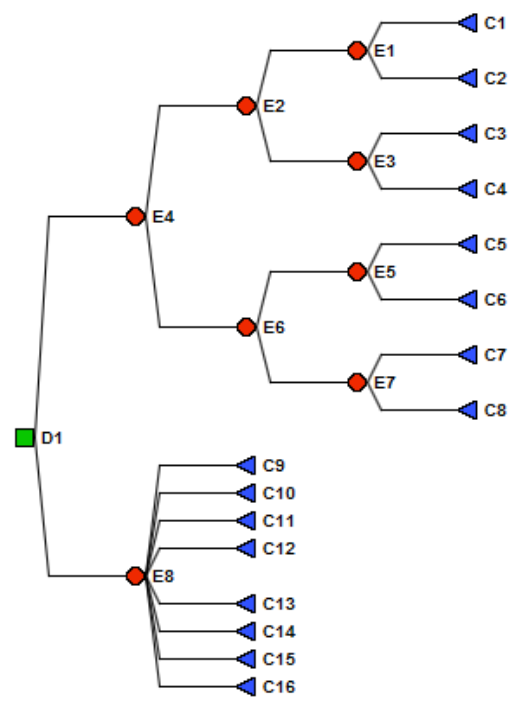

Figure 5. Risk constraint evaluation with contraction analysis (upper) and violation belief analysis (lower) using $(r, s)=(0.1,0.1)$.

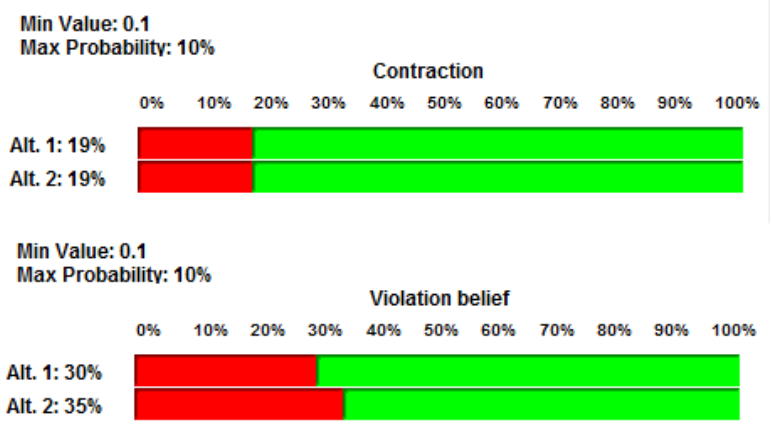

Figure 6. Risk constraints evaluation with contraction analysis (upper) and violation belief analysis (lower) using $(r, s)=(0.1,0.05)$.

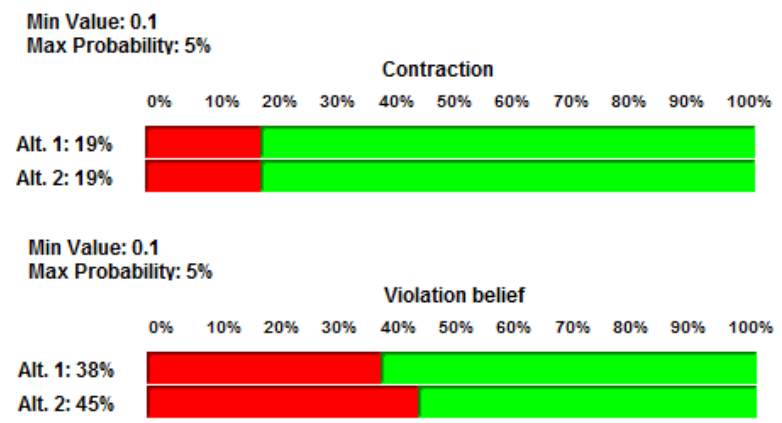


Now assume that we sharpen the risk constraint so that we have $(r, s)=(0.1,0.05)$, i.e., we stipulate a lower probability. The amount of contraction required not to violate the risk constraints is unchanged, while it is changed for the violation belief analysis as a consequence of probability sharpening, See Figures 5 and 6.

\section{Summary and Conclusions}

The various axiomatic systems proposed supporting the principle of maximizing the expected utility are insufficient and have been subject to severe criticism. One criticism is that the classical notion of a utility function cannot cover some quite natural risk behavior, such as not accepting an alternative independently of its expected utility because one or more consequences are too severe regardless of there existing consequences with high utilities and high probabilities to occur, resulting in a beneficial expected utility. Due to these criticisms and from pragmatic issues in employing this principle as a rule for rational choice, it is worthwhile to supplement frameworks based on the utility principle with other decision rules taking a wider spectrum of risk attitudes into account. One such supplement is the inclusion of thresholds in the form of risk constraints.

This paper discusses how numerically imprecise information can be modeled and evaluated with decision trees, and how the risk evaluation process can be elaborated by integrating mechanisms for handling vague and numerically imprecise probabilities and utilities. The shortcomings of the principle of maximizing the expected utility can in part be compensated for by the introduction of the concept of risk constraint violation. It should be emphasized that this is not the only method of comparing the risk involved in different alternatives in imprecise domains. However, it is based on a well-founded model of imprecision and meets reasonable requirements on its properties.

Using the concept of SORC violation, a general model can be constructed for representing various risk attitudes. The definitions are computationally meaningful, and are therefore also well suited to automated decision making. Rules have been suggested for sorting out undesirable decision alternatives, rules which could also serve as a tool for guaranteeing that certain norms are not violated.

\section{Acknowledgments}

This research was funded by the Swedish Research Council FORMAS, project number 2011-3313-20412-31, as well as by Strategic funds from the Swedish government within ICT — The Next Generation.

\section{Conflicts of Interest}

The authors declare no conflicts of interest.

\section{References}

1. Aven, T. Risk Analysis: Assessing Uncertainties Beyond Expected Values and Probabilities; Wiley: Chichester, United Kingdom, 2008. 
2. Gregory, R.S. Valuing Risk Management Choices. In Risk Analysis and Society: An Interdisciplinary Characterization of the Field; McDaniels, T., Small, M., Eds.; Cambridge, United Kingdom, 2004; pp. 213-250.

3. Shahzad, B.; Safvi, S.A. Effective risk mitigation: A user prospective. Int. J. Math. Comput. Simul. 2008, 1, 70-80.

4. Nagashima, T.; Nakamura, K.; Shirakawa, K.; Komiya, S. A proposal of risk identification based on the improved kepner-tregoe program ant its evaluation. Int. J. Syst. Appl. Eng. Dev. 2008, 4, 245-257.

5. Von Neumann, J.; Morgenstern, O. Theory of Games and Economic Behavior; Princeton University Press: Princeton NJ, USA, 1947.

6. Ramsey, F.P. Truth and Probability. In The Foundations of Mathematics and other Logical Essays; Cambridge University Press: Cambridge, UK ; 1931, reprinted in Gardenfors and Sahlin (eds.), Decision, Probability, and Utility, Cambridge University Press: Cambridge, United Kingdom, 1988; pp. 19-47.

7. Savage, L.J. The theory of statistical decision. J. Am. Stat. Assoc. 1951, 46, 55-67.

8. Malmnäs, P-E. Axiomatic justifications of the utility principle: A formal investigation. Synthese 1994, 99, 233-249.

9. Schoemaker, P.J.H. The expected utility model: Its variants, purposes, evidence and limitations. J. Econ. Lit. 1982, 20, 529-563.

10. Loomes, G.; Sugden, R. Regret theory: An alternative theory of rational choice under uncertainty. Econ. J. 1982, 92, 805-824.

11. Malmnäs, P-E. Evaluations, Preferences, Choice Rules; Research Report; Department of Philosophy, Stockholm University: Stockholm, Sweden, 1996.

12. Riabacke, A.; Påhlman, M.; Larsson, A. How different choice strategies can affect the risk elicitation process. IAENG Int. J. Comput. Sci. 2006, 32, 460-465.

13. Riabacke, M.; Danielson, M.; Ekenberg, L. State-of-the-art prescriptive criteria weight elicitation. Adv. Decis. Sci. 2012, 2012, 276584:1-276584:24.

14. Mason, C.F.; Shogren, J.; Settle, C.; List, A.J. Environmental catastrophes and non-expected utility maximization: An experimental evaluation. J. Risk Uncertain. 2005, 31, 187-215.

15. Keeney, R.L. Decision analysis: An overview. Oper. Res. 1982, 30, 803-838.

16. Frohwein, H.I.; Lambert, J.H. Risk of extreme events in multiobjective decision trees Part 1. Severe events. Risk Anal. 2000, 20, 113-123.

17. Haimes, Y.Y. Risk Modeling, Assessment, and Management; Wiley: New York, NY, USA, 1998.

18. Satoh, N.; Kumamoto, H. Analysis of information security problem by probabilistic risk assessment. Int. J. Comput. 2009, 3, 337-347.

19. Danielson, M.; Ekenberg, L. Computing upper and lower bounds in interval decision trees. Eur. J. Oper. Res. 2007, 181, 808-816.

20. Danielson, M.; Ekenberg, L.; Larsson, A. Distribution of expected utility in decision trees. Int. J. Approx. Reason. 2007, 46, 387-407.

21. Alikhani, A. Proposed risk control in strategic management project for Qomrud River in Iran. Int. J. Energy Environ. 2009, 3, 112-121. 
22. Danielson, M. Generalized evaluation in decision analysis. Eur. J. Oper. Res. 2005, 162, 442-449.

23. Ekenberg, L.; Boman, M.; Danielson, M. A tool for coordinating autonomous agents with conflicting goals. In Proceedings of the 1st International Conference on Multi-Agent Systems, San Francisco, CA, USA, 12-14 June 1995; pp. 89-93.

24. Ekenberg, L.; Danielson, M.; Boman, M. Imposing security constraints on agent-based decision support. Decis. Support Syst. 1997, 20, 3-15.

25. Ekenberg, L.; Boman, M.; Linneroth-Bayer, J. General risk constraints. J. Risk Res. 2001, 4, $31-47$.

26. Larsson, A.; Johansson, J.; Ekenberg, L.; Danielson, M. Decision analysis with multiple objectives in a framework for evaluating imprecision. Int. J. Uncertain. Fuzziness Knowl.-Based Syst. 2005, 13, 495-509.

27. Ekenberg, L.; Thorbiörnson, J. Second-order decision analysis. Int. J. Uncertain. Fuzziness Knowl.-Based Syst. 2001, 9, 13-38.

(c) 2014 by the authors; licensee MDPI, Basel, Switzerland. This article is an open access article distributed under the terms and conditions of the Creative Commons Attribution license (http://creativecommons.org/licenses/by/3.0/). 